\title{
A novel mouse model of lipotoxic cardiomyopathy
}

\author{
Hsiu-Chiang Chiu, ${ }^{1}$ Attila Kovacs, ${ }^{1}$ David A. Ford,${ }^{2}$ Fong-Fu Hsu, ${ }^{3}$ Ricardo Garcia, ${ }^{1}$ \\ Pilar Herrero, ${ }^{4}$ Jeffrey E. Saffitz, ${ }^{1,5}$ and Jean E. Schaffer ${ }^{1}$ \\ ${ }^{1}$ Center for Cardiovascular Research, Department of Internal Medicine, Washington University School of Medicine, \\ St. Louis, Missouri, USA \\ ${ }^{2}$ Department of Biochemistry and Molecular Biology, St. Louis University Health Sciences Center, St. Louis, Missouri, USA \\ ${ }^{3}$ Department of Internal Medicine, \\ ${ }^{4}$ Department of Radiology, and \\ ${ }^{5}$ Department of Pathology, Washington University School of Medicine, St. Louis, Missouri, USA \\ Address correspondence to: Jean Schaffer, Washington University School of Medicine, 660 South Euclid Avenue, Box 8086, \\ St. Louis, Missouri 63110, USA. Phone: (314) 362-8717; Fax: (314) 362-0186; E-mail: jschaff@imgate.wustl.edu.
}

Received for publication August 4, 2000, and accepted in revised form February 26, 2001.

Inherited and acquired cardiomyopathies are associated with marked intracellular lipid accumulation in the heart. To test the hypothesis that mismatch between myocardial fatty acid uptake and utilization leads to the accumulation of cardiotoxic lipid species, and to establish a mouse model of metabolic cardiomyopathy, we generated transgenic mouse lines that overexpress long-chain acylCoA synthetase in the heart (MHC-ACS). This protein plays an important role in vectorial fatty acid transport across the plasma membrane. MHC-ACS mice demonstrate cardiac-restricted expression of the transgene and marked cardiac myocyte triglyceride accumulation. Lipid accumulation is associated with initial cardiac hypertrophy, followed by the development of left-ventricular dysfunction and premature death. Terminal deoxynucleotidyl transferase-mediated dUTP nick-end labeling staining and cytochrome $c$ release in transgenic hearts suggest that cardiac myocyte death occurs, in part, by lipid-induced programmed cell death. Taken together, our data demonstrate that fatty acid uptake/utilization mismatch in the heart leads to accumulation of lipid species toxic to cardiac myocytes. This novel mouse model will provide insight into the role of perturbations in myocardial lipid metabolism in the pathogenesis of inherited and acquired forms of heart failure.

J. Clin. Invest. 107:813-822 (2001).

\section{Introduction}

Under normal physiologic conditions, cardiac myocytes in the postnatal mammalian heart rely on $\beta$-oxidation of long-chain fatty acids (LCFAs) to generate ATP (1). Since these cells have little capacity for de novo biosynthesis or storage of LCFAs (2) and since serum unbound FFA concentrations are in the low nanomolar range (3), cardiac myocytes require an efficient mechanism for importing this metabolic substrate. At physiologic concentrations of LCFAs, uptake by isolated cardiac myocytes is saturable, temperature dependent, and inhibited by previous protease treatment, suggesting that sarcolemmal proteins mediate LCFA permeation (4-6).

Several proteins have been shown to facilitate movement of LCFAs across the plasma membrane of mammalian cells, including the fatty acid transport protein (FATP1) and long-chain acyl-CoA synthetase (ACS1) (7-10). ACS1 is highly expressed in the heart and catalyzes esterification of LCFAs with coenzyme A (CoA), the initial step in fatty acid metabolism. ACS1 has broad substrate specificity, catalyzing esterification of saturated fatty acids with 10-18 carbons and monoand polyunsaturated fatty acid with 16-20 carbons (11). In addition to association with intracellular membranes, ACS1 is found at the plasma membrane of cultured adipocytes, where it may function coordinately with FATP1 to facilitate vectorial LCFA uptake by esterifying LCFAs immediately upon transport (10).

In a number of pathophysiologic states, evidence is emerging that mismatch between uptake and utilization of LCFAs leads to abnormally high intracellular LCFA concentrations. First, myocardial metabolism switches from utilization of LCFAs to utilization of glucose during the development of cardiac hypertrophy and in the ischemic and failing heart (12-16). While this metabolic switch may initially serve an adaptive function, accumulation of intracellular LCFAs in these acquired conditions has been proposed to contribute to contractile dysfunction and the generation of cardiac arrhythmias (17). Second, increased cardiac myocyte triglyceride stores are observed in animal models of obesity and in diabetes, disease states in which high serum FFA levels are thought to promote LCFA uptake in excess of tissue capacity for utilization (18-20). This lipid accumulation has been proposed to contribute to cardiac myocyte apoptosis and congestive heart failure $(18,21-23)$. Third, inherited defects in the mitochondrial fatty acid oxidation pathway have been associated with cardiomyopathy and sudden death in children and young adults (24). Postmortem pathologic studies demonstrate marked intracellular lipid accumulation in the heart, the result of persistent LCFA import in the 
face of blocked LCFA metabolism (25). It remains unclear whether the cardiomyopathy in these pathologic states results from toxic lipid accumulation or whether cardiomyopathy results directly from altered myocyte metabolism (e.g., energy starvation).

The present study was designed to test the hypothesis that mismatch between lipid import and capacity for lipid utilization leads to cardiotoxic lipid accumulation and resultant heart failure. We generated transgenic mouse lines with cardiac-restricted overexpression of ACS1. These mice demonstrate marked cardiac myocyte triglyceride accumulation and develop cardiomyopathy. This study provides insight into the role of perturbations of myocardial lipid metabolism in the pathogenesis of inherited and acquired forms of heart failure.

\section{Methods}

Transgenic mice. An $\alpha-\mathrm{MHC}$ promoter construct containing the last exon of the $\beta$-MHC gene, the $\alpha-\mathrm{MHC}$ promoter, the first three untranslated exons (and two introns) of the $\alpha$-MHC gene, a SalI to HindIII cloning site, and polyadenylation sequences from the human growth hormone gene was obtained from J. Robbins (University of Cincinnati, Cincinnati, Ohio, USA) (26). The murine adipocyte ACS1 cDNA (9) was cloned into the SalI site with a MYC epitope tag (MEQKLISEEDLN) and glycine/glycine liner inserted after the initiator methionine. A linearized 8.1-kb NotI fragment of the construct containing the promoter, introns, cDNA, and polyadenylation sequences was isolated, purified, and microinjected into $\mathrm{FVB} / \mathrm{N}$ mouse embryos that were implanted into pseudopregnant CBA/B6 foster mothers. Screening for founders was performed by PCR analysis of tail DNA using the following primers: 5'-ATAAGCGAAGAAGATCTTAATGGAGG- $3^{\prime}$ and $5^{\prime}$-TTTGTTCACTATGTAGGTGATGGCCT$3^{\prime}$, which generate a transgene-specific fragment of 605 bp. As a control, coamplification of a 289-bp fragment of the endogenous intestinal fatty acid-binding protein gene was performed. Integration of the transgene was confirmed by Southern blot analysis of tail DNA using transgene-specific human growth hormone polyadenylation sequences as probe. Founder animals were propagated by breeding with FVB/N wild-type mice. Mice were maintained on a $5 \%$ fat chow diet.

Western blot analysis for ACS and transgene expression. Ventricular tissue (free of atrial and vascular structures) or other organs (brain, liver, skeletal muscle, and fat) from 18- to 30-day-old transgenic mice and age-matched control littermates was snap-frozen in liquid nitrogen. Tissues were disrupted by 40 strokes in a glass-Teflon homogenizer in buffer containing $20 \mathrm{mM}$ Tris (pH 7.4), $1 \mathrm{mM}$ EDTA, $255 \mathrm{mM}$ sucrose, $1 \mathrm{mM}$ PMSF, and $1 \times$ Protease Complete (Boehringer Mannheim, Indianapolis, Indiana, USA). Nuclei were pelleted by centrifugation at $1,000 \mathrm{~g}$ and discarded. Total postnuclear membranes were pelleted by centrifugation at $356,000 \mathrm{~g}$ and resuspended in $1 \% \mathrm{SDS}, 150 \mathrm{mM} \mathrm{NaCl}, 50 \mathrm{mM}$ Tris (pH 7.4), $2 \mathrm{mM}$ EDTA, $1 \mathrm{mM}$ PMSF, $1 \times$ Protease Complete. Pro- teins were quantified by bicinchoninic acid (BCA) assay (Pierce Chemical Co., Rockford, Illinois, USA) and separated by $7.5 \%$ SDS-PAGE. Western blot analysis for total ACS1 expression was performed using rabbit polyclonal antisera directed against ACS1 $(10)(1: 1,000)$ and for transgene-specific protein using a monoclonal antiserum directed against the MYC epitope (sc-40, 1:2000; Santa Cruz Biotechnology, Santa Cruz, California, USA). Detection was performed using a horseradish peroxidase-coupled (HRP-coupled) anti-rabbit Ab (Jackson ImmunoResearch Laboratories Inc., West Grove, Pennsylvania, USA) and Renaissance Western blot chemiluminescence reagents (NEN Life Science Products Inc., Boston, Massachusetts, USA). ACS1-specific signal was quantified using Molecular Analyst Software (Bio-Rad Laboratories Inc., Hercules, California, USA).

Northern analysis. RNA was isolated from snap-frozen hearts of 21-day-old transgenic and control animals using RNeasy midi columns (QIAGEN Inc., Valencia, California, USA) and analyzed by Northern blotting using phospholamban, atrial natriuretic factor (ANF), sarcoplasmic reticulum calcium ATPase (SERCA), and $\alpha$-skeletal actin as probes.

Echocardiography. Longitudinal noninvasive transthoracic echocardiograms were performed as described previously (27). A total of 40 mice were studied on a weekly or biweekly basis (07 line: five transgenic, five wild-type; M13 line: eight transgenic, five wild-type; J3 line: ten transgenic, six wild-type). Digitally acquired echocardiographic images were analyzed off-line by a single observer blinded to the genotype of the animals.

Histology. Ventricular structures were rapidly isolated from sacrificed mice (carbon dioxide asphyxiation). Tissues were fixed overnight in 10\% formalin and paraffinembedded sections were stained with hematoxylin and eosin (H\&E) or Masson's trichrome. Tissues were also snap-frozen, and sections were stained with oil red $O$. Bright-field microscopy was performed using a Zeiss Axioskop microscope, and digital images were obtained on a Spot camera (Advanced Spot Software; Diagnostic Instruments Inc., Sterling Heights, Michigan, USA). Morphometric analysis of H\&E-stained tissues was performed using Zeiss Image software at $\times 400$. We measured cross-sectional area of five randomly chosen cells in each of eight randomly chosen fields from sections from two animals in each group (transgenic and wild-type).

Electron microscopy. Tissue was fixed in glutaraldehyde, post fixed in osmium tetroxide, and embedded in epoxy resin. Ultra-thin sections were examined by transmission electron microscopy (EM).

Serum analysis. After a 4-hour fast, blood was obtained from lightly anesthetized 20-day-old mice by retroorbital bleed. Serum was separated and analyzed for triglycerides (Sigma 339-10), cholesterol (352-20; Sigma Chemical Co., St. Louis, Missouri, USA), glucose (315-100; Sigma Chemical Co.), and FFAs (994-75409; Wako Chemicals USA Inc., Richmond, Virginia, USA).

Tissue lipid analysis. Ventricular structures were rapidly isolated from sacrificed mice and snap-frozen. Tis- 
sue was homogenized and lipids isolated by extraction into chloroform (28) with appropriate internal standards included for each protocol. Chloroform extracts were used for analysis of individual phospholipid molecular species using a Finnigan-MAT TSQ-7000 triple-stage quadrupole mass spectrometer as described previously (29). Electrospray-ionization tandem mass spectrometry was used to substantiate individual molecular species. Additionally, chloroform extracts were separated by thin-layer chromatography (silica gel $G$ plates and a mobile phase of petroleum ether/ethyl ether/acetic acid, 90/10/1, vol/vol/vol) to isolate cholesteryl esters and triacylglycerols. Following acid methanolysis, capillary gas chromatography was performed (Hewlett-Packard 5890; Hewlett-Packard, Palo Alto, California, USA) with a flame-ionization detector using a $40 \mathrm{~m}$ Supelco SP2330 capillary column and a flow rate of $2 \mathrm{~cm}^{3} /$ minute at 20 psi capillary pressure with a 20:1 split ratio. Ceramide was measured from 1 mg of heart tissue by the method of Van Veldoven (30), using diacylglycerol kinase to quantitatively convert ceramides to ${ }^{32} \mathrm{P}$-labeled ceramide phosphates, which are separated from other lipids by thin-layer chromatography (silica gel $G$ plates, mobile phase comprised of chloroform/acetone/methanol/acetic acid/water, $10 / 4 / 3 / 2 / 1, \mathrm{vol} / \mathrm{vol} / \mathrm{vol} / \mathrm{vol} / \mathrm{vol})$. Tissue ceramides were quantified by comparison to a linear ceramide standard curve. All values for lipid analysis are presented as the mean \pm SE. Statistical evaluation between two groups was by student's $t$ test.

Apoptosis studies. Formalin-fixed paraffin-embedded tissues from euthanized 18-day-old transgenic and nontransgenic mice were evaluated by terminal deoxynucleotidyl transferase-mediated dUTP nickend labeling (TUNEL) staining according to manufacturer's specifications (Oncogene Research Products, San Diego, California, USA) with heat treatment $\left(100^{\circ} \mathrm{C}\right)$ for 30 minutes in $1 \mathrm{mM}$ EDTA, pH 8.0, in lieu of proteinase $\mathrm{K}$ digestion. Tissue was also stained with a mAb directed against $\alpha$-sarcomeric actin (A2172 clone 5C5, 1:200; Sigma Chemical Co.) to stain cardiac myocytes with detection using alkaline-phosphatase-coupled anti-mouse IgG, 5-bromo-4-chloro3 -indolylphosphate-4-toluidine, and nitro blue tetrazolium chloride. For analysis of mitochondrial cytochrome $c$ release, snap-frozen tissues from 21-dayold mice were disrupted and fractionated as described previously (31). Protein in soluble and pellet (mitochondria) fractions was quantified by using BCA assay (Pierce Chemical Co.). Twenty micrograms of mitochondrial fraction protein and $80 \mu \mathrm{g}$ of soluble-fraction protein were separated by $15 \%$ SDS-PAGE and analyzed by Western blot using a mouse $\mathrm{mAb}$ directed against cytochrome $c$ (PharMingen 65981A 1:500 dilution; BD PharMingen, San Diego, California, USA) or a mouse $\mathrm{mAb}$ directed against cytochrome oxidase subunit IV (Molecular Probes A-6431, 1:2000 dilution; Molecular Probes Inc., Eugene, Oregon, USA), HRPcoupled anti-mouse $\mathrm{Ab}$, and chemiluminescence.

\section{Results}

Generation of MHC-ACS mice. To create mismatch between cardiac fatty acid import and utilization, we overexpressed ACS1 in the postnatal ventricles of mice using the $\alpha$ myosin heavy-chain gene promoter (26). At the amino terminus of ACS1, we inserted a MYC epitope tag that does not interfere with expression, subcellular location, or function of ACS1 (data not shown). Founders were screened by PCR and Southern blot analysis (data not shown). We characterized three independent MHC-ACS lines and analyzed the $\mathrm{F}_{1}$ and $\mathrm{F}_{2}$ generations. The average litter size was nine pups, and there was no evidence that the transgene resulted in a developmentally lethal phenotype. Expression of the transgene was evaluated by Western blot analysis of membrane proteins from various tissues of 18-dayold animals. MHC-ACS mice had high expression levels of the transgene in the heart with no detectable expression in brain, liver, skeletal muscle, intestine, or fat (Figure 1a). In three independent lines, we observed 6-fold (J3), 9-fold (M13), and 11-fold (O7) increases in ACS1 protein compared with wild-type mice (Figure
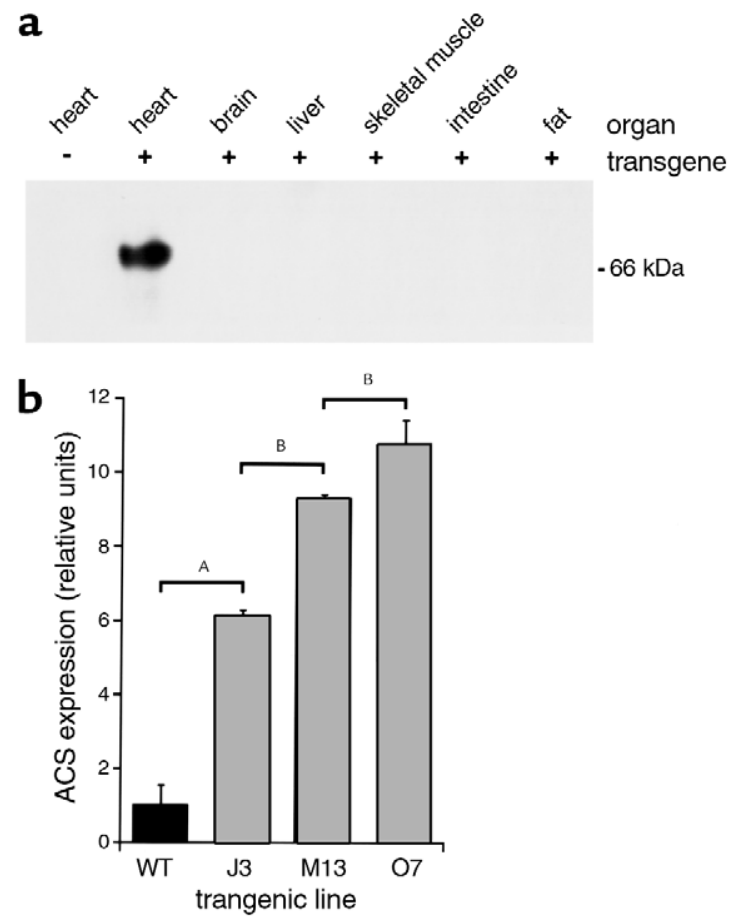

\section{Figure 1}

Cardiac overexpression of murine ACS1 in MHC-ACS lines. (a) Membrane protein $(20 \mu \mathrm{g})$ from various organs of an MHC-ACS O7 transgenic animal and nontransgenic littermate were separated by SDS-PAGE. Specific transgene expression was analyzed by Western blot, using a monoclonal anti-MYC Ab. (b) Membrane protein $(20 \mu \mathrm{g})$ from hearts of 18-day-old MHC-ACS transgenic mice were analyzed by Western blot using rabbit polyclonal antisera directed against native murine ACS1 sequences. ACS1-specific signal was quantified and relative units of ACS1 expression is shown for wildtype (WT) and three independent transgenic lines (J3, M13, and O7). Data are reported as the mean $\pm \mathrm{SE}$. Differences among groups were compared by one-way ANOVA in conjunction with the post hoc Scheffé test $\left({ }^{A} P<0.0001 ;{ }^{B} P<0.01\right)$. 


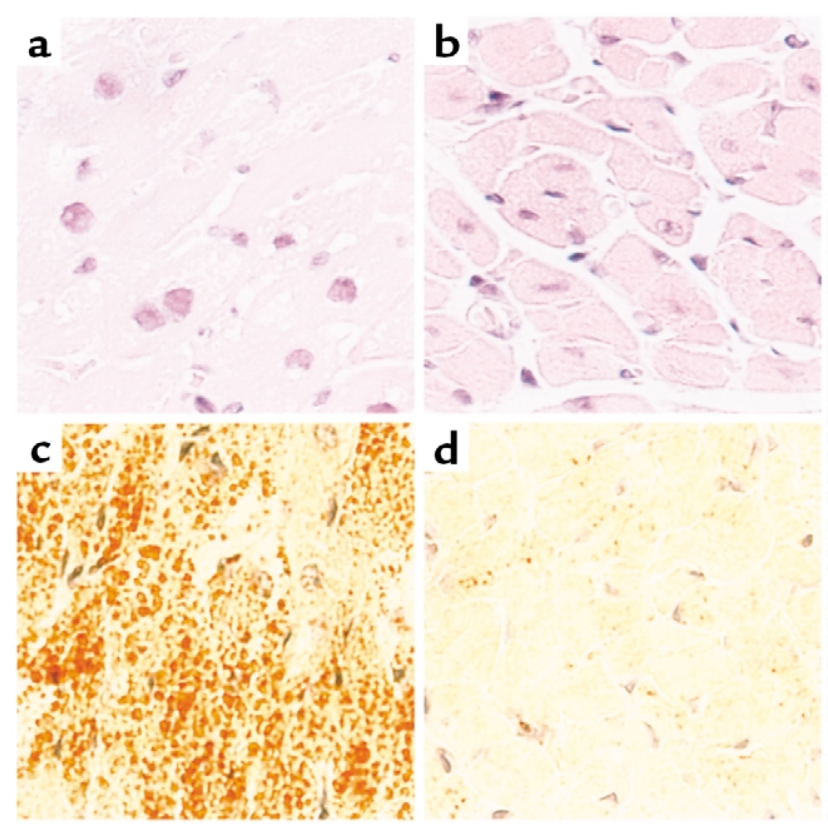

Figure 2

Light-microscopic examination of cardiac tissue from 18-day-old MHC-ACS mice. Cardiac ventricular tissue was dissected from 18-dayold MHC-ACS O7 animals (a, c) and nontransgenic littermates (b, d). Tissue was fixed in formalin, embedded in paraffin, and sectioned for $\mathrm{H} \& \mathrm{E}$ staining $(\mathbf{a}, \mathbf{b})$. Tissue was flash-frozen and sectioned for oil red $O$ staining $(\mathbf{c}, \mathbf{d}) . \times 400$.

1b). The transgene dose as determined by Southern blot analysis correlated with these graded levels of expression (data not shown).

Lipid accumulation in cardiac myocytes of MHC-ACS mice. Histologic analyses were used to assess qualitatively the effects ACS1 overexpression on myocardial lipid content in 18-day-old O7 mice. H\&E staining of transgenic hearts revealed vacuolated myocytes, consistent with intracellular accumulation of lipid (Figure 2, a and b). Oil red O staining showed numerous small droplets of neutral lipid throughout the cytosol of transgenic myocytes (Figure 2, c and d). EM demonstrated relatively preserved cytoarchitecture with abundant cytosolic lipid droplets, corresponding to neutral lipid observed by oil red $\mathrm{O}$ and vacuoles by $\mathrm{H} \& \mathrm{E}$ staining (Figure 3 ). A spectrum of droplet structures was observed that included amorphous droplets of homogeneous electron density without apparent limiting membrane, droplets with occasional electron-dense linear streaks, and droplets surrounded by a single limiting membrane or multiple concentric membrane lamellae.

We used gas chromatography and mass spectrometry to identify and quantify individual lipid species in extracts from 18-day-old O7 transgenic hearts (Figure 4). Transgenic hearts had a 12-fold increase in triglyceride content with broad acyl-chain distribution (C14 to C18), including both saturated and unsaturated species. In contrast there was no change in content of another neutral lipid, cholesteryl ester. We observed a 50\% increase in choline glycerophospholipids and a $15 \%$ increase in ethanolamine glycerophospholipids. These findings suggest that the structures observed by oil red $\mathrm{O}$ staining and EM consist primarily of triglycerides. The smaller increase in choline glycerophospholipids may be attributable to the membranous structures surrounding the lipid droplets. For both choline and ethanolamine glycerophospholipids there was a shift in fatty acid composition at the SN-2 position, with increases in $18: 1$, $18: 2$, and 20:4 species and decreases in 20:6. The shift in acyl-chain composition may result from the substrate specificity of the ACS1 isoform used in these experiments. The changes in cardiac lipid content occurred in the setting of normal serum fatty acid, triglyceride, glucose, and cholesterol (data not shown). No changes in FFAs or acyl-carnitines were observed in transgenic hearts (data not shown).

Heart failure in MHC-ACS mice. Although MHC-ACS mice appeared normal at birth, they displayed significant morbidity and mortality. As early as 4 weeks of age, MHCACS mice developed overt heart failure, characterized by lethargy, shortness of breath, and cyanosis. Many spontaneous deaths occurred in the MHC-ACS lines, whereas no deaths were observed in wild-type age- and sexmatched littermates (Figure 5a). The incidence of heart failure or death was highest in the MHC-ACS O7 line. All transgenic offspring from the $\mathrm{O} 7$ line and many from the M13 line died by 100 days. To propagate these lines, we transplanted the ovaries from transgenic M13 and O7 females into nontransgenic recipients. Rare deaths were

\footnotetext{
Figure 3

EM of cardiac tissue from 18-day-old MHC-ACS mice. Ultra-thin sections of fixed ventricular tissue from 18-day-old MHC-ACS O7 animals were examined by transmission EM. Images are shown at $\times 7,500(\mathbf{a}$; bar, $1 \mu \mathrm{m})$, and at $\times 15,000(\mathbf{b}$; bar, $0.5 \mu \mathrm{m})$. Numerous lipid droplets (L) are observed in ventricular myocytes. Some droplets at this stage are surrounded by multiple concentric layers of membrane (arrow).
}
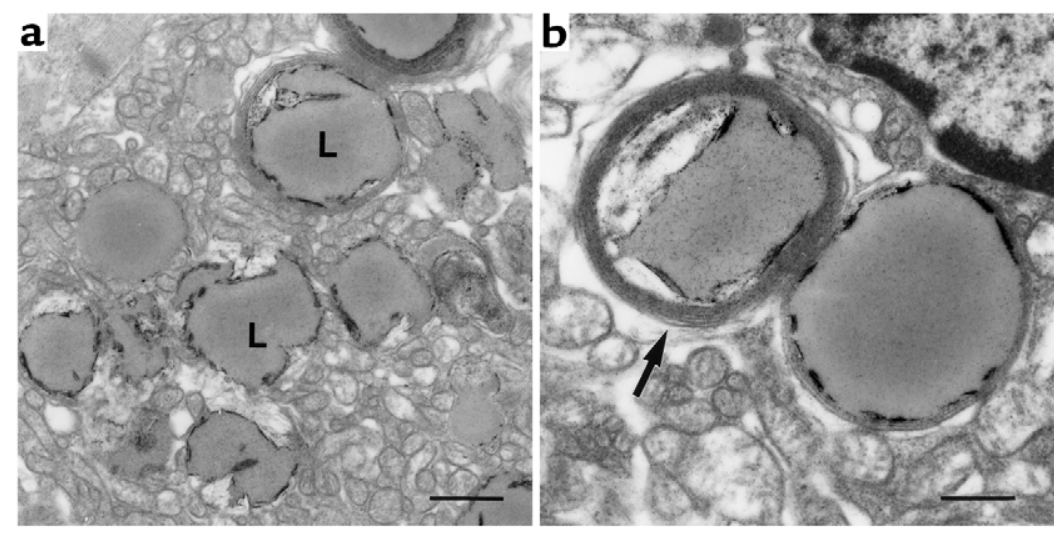
Figure 4

Cardiac lipid accumulation in 18-day-old MHC-ACS mice. Lipids were extracted from frozen ventricular tissues of wild-type and transgenic (O7) mice. Triacylglycerols (a), cholesteryl esters (b), choline glycerophospholipids (c), and ethanolamine glycerophospholipids (d), were quantified. Data from wild-type and transgenic mice are displayed with filled and open bars, respectively. Data for each measurement report the mean of a minimum of five animals \pm SE. Statistical evaluation between groups was by Student's $t$ test ( $\left.{ }^{A} P<0.01\right)$. ND, not detected; $P$, plasmalogen species. a

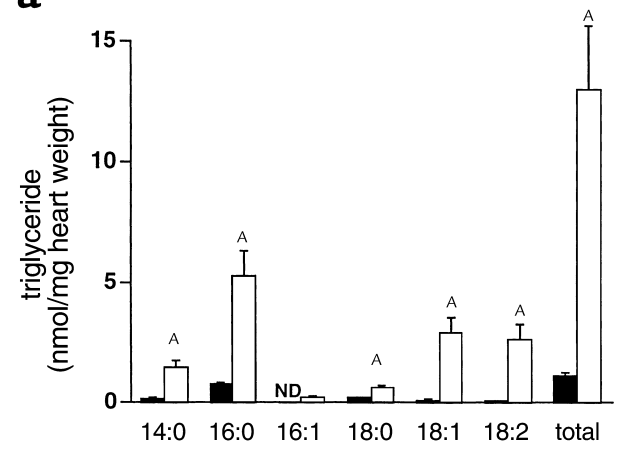

C

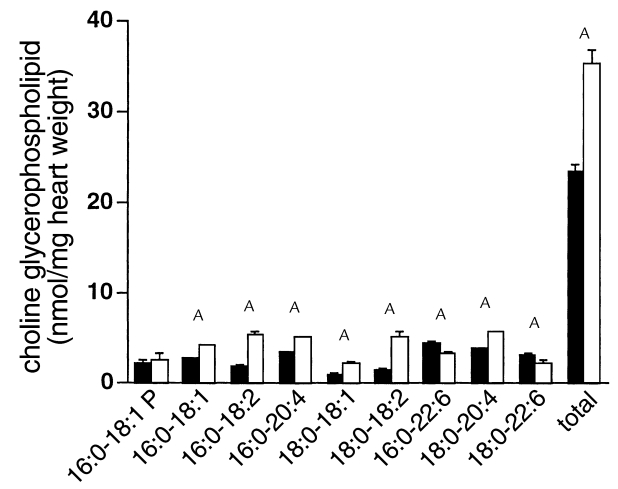

b

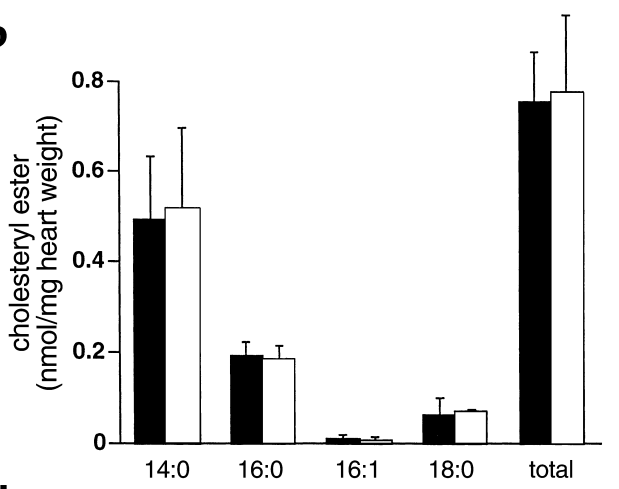

d

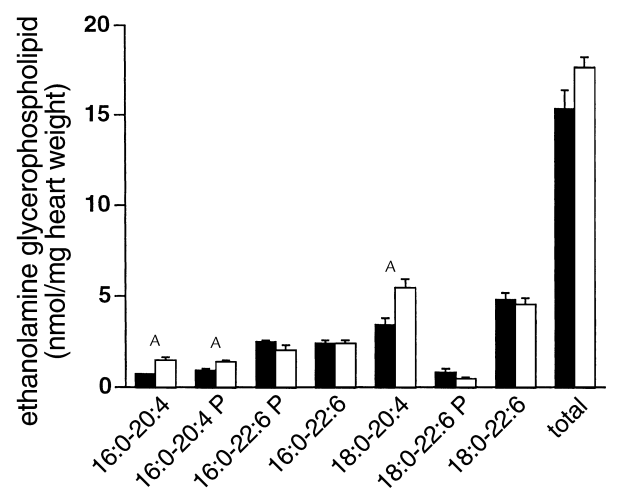

observed in the J3 line, which has a lower level of transgene expression. Survival was thus inversely related to the level of transgene expression, and the lower level of expression in J3 mice appears to be below a threshold for development of early increased morbidity or mortality. In all three MHC-ACS lines, at the time of death or sacrifice, heart weight relative to body weight was increased compared with age- and sex-matched nontransgenic littermates (Figure 5b). The degree of cardiomegaly correlated with the level of transgene expression (least in J3 line, most in $\mathrm{O} 7$ line), suggesting a dose-response relationship.

To further evaluate the structural and functional cardiac phenotype in MHC-ACS mice, we performed weekly transthoracic echocardiograms. Figure 6a shows representative two-dimensional guided $\mathrm{M}$-mode images of the left ventricle. High-quality $\mathrm{M}$-mode recordings were obtained even at 2 weeks of age when the average body weight of wild-type mice is $9 \mathrm{~g}$ and mean left-ventricular

\section{Figure 5}

Premature death and cardiomegaly in MHC-ACS mice. (a) Wild-type (WT, $n=110)$ and transgenic $(\mathrm{J} 3, n=19 ; \mathrm{M} 13, n=23 ; \mathrm{O} 7, n=9)$ mice were followed for 130 days, and incidence of spontaneous death was recorded as a function of time. Differences among survival curves were compared using the log-rank test $\left({ }^{A} P<0.05,{ }^{B} P<0.01,{ }^{C} P<0.0001\right)$. (b) At the time of spontaneous death or sacrifice, mice were weighed. Hearts were dissected, and atrial and vascular structures were removed before weighing. Heart-to-body weight ratios were determined in wild-type (solid bars) and transgenic (open bars for J3, M13, O7) mice older than 24 days at the time of death or sacrifice. For each transgenic mouse, an age- and sex-matched wild-type littermate was sacrificed and analyzed. Differences among groups were compared by two-way ANOVA $\left({ }^{A} P<0.0001\right)$. mass of wild-type mice is $20 \mathrm{mg}$. Echocardiographic data from $\mathrm{O} 7$ mice is summarized in Table 1. Compared with the normal growth of the wild-type left ventricle over the first 2 months of life, we observed significantly increased left-ventricular mass developing at 3 weeks of age and progressively increasing over time in the $\mathrm{O} 7$ and M13 lines (Figure 6b). In contrast, left-ventricular mass measurements in transgenic line J3 showed development of more modest hypertrophy reaching significance at 5
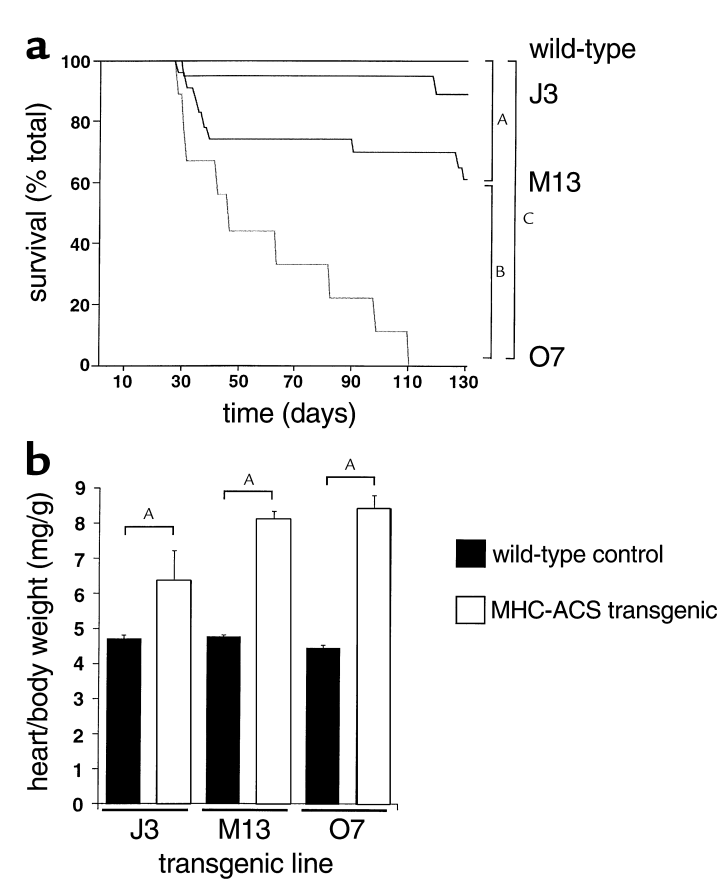
Figure 6

Cardiac hypertrophy and failure in MHC-ACS mice. (a) Two-dimensional guided M-mode echocardiographic images were obtained from wild-type (upper panel) and MHC-ACS1 O7 (lower panel) mice at 2, 3, 4, and 6 weeks of age. Images shown are from a representative pair of age- and sex-matched wild-type and transgenic animals. (b) Quantification of left-ventricular (LV) mass was performed on age- and sex-matched wild-type and transgenic (J3, M13, O7) mice based on serial echocardiograms at the indicated time points. A minimum of five different animals were examined at each time point. Data points are expressed as mean $\pm \mathrm{SD}$. $A, B, C P<0.05$ for $\mathrm{O} 7$, $\mathrm{M} 13$, or J3, respectively, versus wild-type. (c) Quantification of fractional shortening over time as assessed by serial echocardiograms for age- and sex-matched wild-type and transgenic mice (J3, M13, O7). Data points are expressed as mean \pm SD. ${ }^{A, B}, C P<0.05$ for $\mathrm{O} 7, \mathrm{M} 13$, or J3, respectively, versus wild-type.
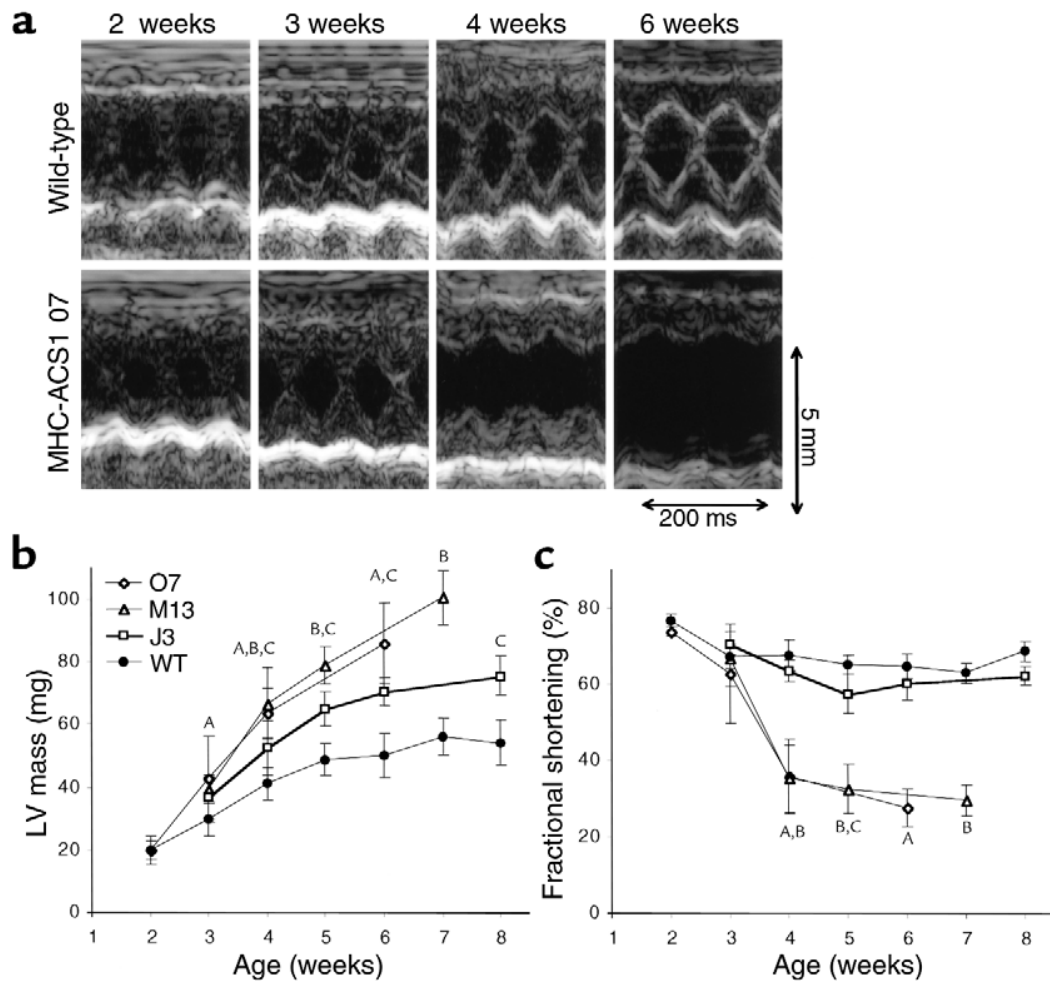

weeks of age. Evaluation of left-ventricular systolic function in lines $\mathrm{O} 7$ and M13 revealed normal fractional shortening at 2 and 3 weeks of age and marked deterioration of systolic performance from 4 weeks onward (Figure 6 , a and c). In contrast, line $\mathrm{J} 3$ animals had preserved systolic function throughout the study period except at 5 weeks when fractional shortening was slightly but significantly decreased compared with wild-type littermates. Preliminary studies of older $\mathrm{J} 3$ mice (4 months) reveal significantly decreased left-ventricular systolic function (data not shown), similar to findings in younger $\mathrm{O} 7$ and M13 mice. Relative wall thickness (a weight-independent parameter of concentric versus eccentric form of left-ventricular hypertrophy) was increased at 3 weeks and subsequently decreased below controls in lines $\mathrm{O} 7$ and M13, but remained increased in line J3 (data not shown). Thus, transgenic mice with highest levels of transgene expression develop concentric left-ventricular hypertrophy with preserved systolic function at an early age (3 weeks), fol- lowed by a transition to eccentric hypertrophy and marked reduction of systolic function. Transgenic mice with lower-level transgene expression develop a concentric form of left-ventricular hypertrophy, maintain near normal systolic function in the first 2 months of life, and subsequently develop heart failure.

To explore potential mechanisms in the development of this lipid-induced cardiomyopathy, we examined ventricular tissue from $\mathrm{O} 7$ transgenic mice between 18 and 21 days of age when there is echocardiographic evidence of hypertrophy, but systolic function remains intact. Morphometric analysis of H\&E-stained tissue showed a $54 \%$ increase in cell size in transgenic mice compared with controls $\left(2.2 \pm 0.08 \times 10^{-4} \mathrm{~mm}^{2}\right.$ in transgenic animals compared with $1.43 \pm 0.03 \times 10^{-4} \mathrm{~mm}^{2}$ in wild-type animals), consistent with myocyte hypertrophy. Northern analysis of mRNA revealed 64\% increase in ANF expression in hearts of transgenic mice (data not shown). However, we observed no changes in other

Table 1

Echocardiographic assessment of left ventricular geometry and systolic function in ACS 07 transgenic line

\begin{tabular}{lcccccccc}
\cline { 2 - 4 } & \multicolumn{2}{c}{2 weeks } & \multicolumn{2}{c}{3 weeks } & \multicolumn{2}{c}{4 weeks } & \multicolumn{2}{c}{6 weeks } \\
& WT & TG & WT & TG & WT & TG & WT & 7 \\
$n$ & 3 & 6 & 6 & 9 & 9 & 11 & 7 \\
HR & $661 \pm 56$ & $655 \pm 28$ & $684 \pm 27$ & $599 \pm 62^{\mathrm{A}}$ & $691 \pm 48$ & $544 \pm 106^{\mathrm{A}}$ & $693 \pm 32$ & $587 \pm 52^{\mathrm{A}}$ \\
LVPWd & $0.43 \pm 0.03$ & $0.44 \pm 0.05$ & $0.45 \pm 0.05$ & $0.54 \pm 0.05^{\mathrm{A}}$ & $0.55 \pm 0.04$ & $0.59 \pm 0.03^{\mathrm{A}}$ & $0.60 \pm 0.03$ & $0.59 \pm 0.06$ \\
LVIDd & $2.3 \pm 0.2$ & $2.2 \pm 0.2$ & $2.6 \pm 0.2$ & $2.9 \pm 0.4$ & $2.8 \pm 0.2$ & $3.5 \pm 0.3^{\mathrm{A}}$ & $2.9 \pm 0.2$ & $4.1 \pm 0.4^{\mathrm{A}}$ \\
LVM & $20 \pm 3$ & $20 \pm 5$ & $28 \pm 5$ & $43 \pm 14^{\mathrm{A}}$ & $41 \pm 6$ & $64 \pm 8^{\mathrm{A}}$ & $49 \pm 5$ & $86 \pm 13^{\mathrm{A}}$ \\
RWT & $0.39 \pm 0.05$ & $0.41 \pm 0.03$ & $0.36 \pm 0.04$ & $0.38 \pm 0.04$ & $0.40 \pm 0.04$ & $0.36 \pm 0.04^{\mathrm{A}}$ & $0.43 \pm 0.03$ & $0.30 \pm 0.05^{\mathrm{A}}$ \\
FS & $77 \pm 2$ & $74 \pm 1^{\mathrm{A}}$ & $66 \pm 3$ & $63 \pm 13$ & $66 \pm 3$ & $36 \pm 10^{\mathrm{A}}$ & $65 \pm 3$ & $28 \pm 5^{\mathrm{A}}$
\end{tabular}

WT, wild-type control; TG, transgenic line; HR, heart rate; LVPWd, left ventricular posterior wall thickness in end diastole; LVIDd, left ventricular internal dimension in end diastole; LVM, left ventricular mass; RWT, relative wall thickness ( $2 \times$ LVPWd/LVIDd); FS, fractional shortening [(LVIDd - LVIDs)/LVIDd]. AP > 0.05. 
markers of myocardial hypertrophy such as phospholamban, $\alpha$-skeletal actin, or SERCA, suggesting that the increase in left-ventricular mass results from accumulation of intracellular lipid rather than from a lipidinduced hypertrophic growth program.

Since cellular lipid accumulation has been shown in other systems to trigger programmed cell death, we also examined ventricular tissue for evidence of apoptosis. In 18-day-old O7 transgenic hearts approximately $1 / 10^{4}$ cells showed evidence of DNA fragmentation by TUNEL, whereas no TUNEL-positive cells were observed in nontransgenic controls (Figure $7, \mathrm{a}-\mathrm{c}$ ). The presence of striations and costaining for $\alpha$-sarcomeric actin suggest that these TUNEL-positive cells are myocytes. In addition, the hearts of transgenic mice demonstrated significant cytochrome $c$ release from mitochondria (Figure 7d). Because fatty acid accumulation in cultured cells has been shown to increase synthesis of ceramide, a lipid capable of inducing apoptosis, we measured ceramide content in 18-day-old transgenic and wild-type hearts using the diacylglycerol kinase assay. Compared with nontransgenic animals, transgenic mice demonstrated a 3.3-fold increase in total cardiac ceramide content (Fig- ure 7e). TUNEL-positive myocytes, cytochrome $c$ release, and increased ceramide content are findings consistent with stimulation of apoptotic pathways. Each of these findings was observed at a time after lipid accumulation occurred and before significant left-ventricular dysfunction, suggesting that lipid-induced programmed cell death contributes to the development of cardiomyopathy in MHC-ACS mice.

We observed marked cardiac myocyte cell loss and evidence for lipid remodeling in end-stage hearts from 28day-old MHC-ACS O7 animals with end-stage heart failure. $H \& E$ staining revealed vacuolated cytoplasm in hypertrophied cardiac myocytes (Figure 8, a and b). Focal mononuclear infiltrate and interstitial fibrosis were observed (Figure 8, e and f). Focal areas of replacement fibrosis were also seen. These observations suggest that necrotic as well as apoptotic cell loss had occurred. Compared with cardiac tissue from 18-day-old animals, 28-dayold mice showed oil red $\mathrm{O}$ staining in only some myocytes (Figure 8, $c$ and d). Together, these findings suggest that cells containing large amounts of triglyceride in 18-dayold mice are selectively lost, contributing to the evolution of heart failure. It is also possible that surviving cells in the

\section{Figure 7}

Evidence for lipoapoptosis in MHC-ACS hearts. (a-c) Cardiac ventricular tissues from a 18-dayold $\mathrm{O} 7$ transgenic mice ( $\mathbf{a}$ and $\mathbf{c}$ ) and from a nontransgenic control littermate (b) were fixed in formalin, embedded in paraffin, and sectioned. Tissues were stained for DNA fragmentation by a TUNEL protocol that stains apoptotic nuclei brown and allows visualization of myocyte striations. Double staining for $\alpha$-sarcomeric actin was used to identify cardiomyocytes in c (myocyte cytoplasm blue). (d) Cardiac ventricular tissues from 21-day-old O7 transgenic mice and nontransgenic control littermates were flash-frozen, homogenized, and separated by differential density centrifugation to yield a membrane fraction (mitochondria) and soluble fraction (cytosol). Membrane protein (20 $\mu \mathrm{g})$ and soluble protein $(80 \mu \mathrm{g})$ were separated by SDS-PAGE and analyzed by Western blotting using an anti-cytochrome $c$ (cyt $c$ ) $\mathrm{Ab}$ and an anti-cytochrome oxidase subunit IV (cyt ox IV) Ab. Bands were quantified using Molecular Analyst software, and relative units of expression are shown for wild-type (-) and transgenic $(+)$ tissues. Data points are expressed as mean (minimum of four independent samples) \pm SE. Nonpaired $t$ test was used to compare groups $\left({ }^{A} P=0.06,{ }^{B} P=0.01,{ }^{C} P<0.01\right)$. (e) Ceramide was measured in heart tissue from 18-day-old transgenic and wild-type animals using the diacylglycerol kinase assay and normalized for tissue weight. Data points are expressed as mean (minimum of five independent samples) \pm SE. Statistical evaluation between groups was by Student's $t$ test (AP $<0.0005)$. a

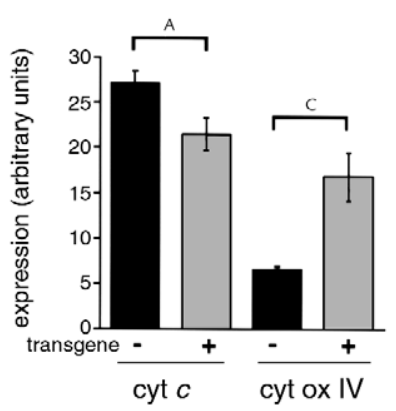

e
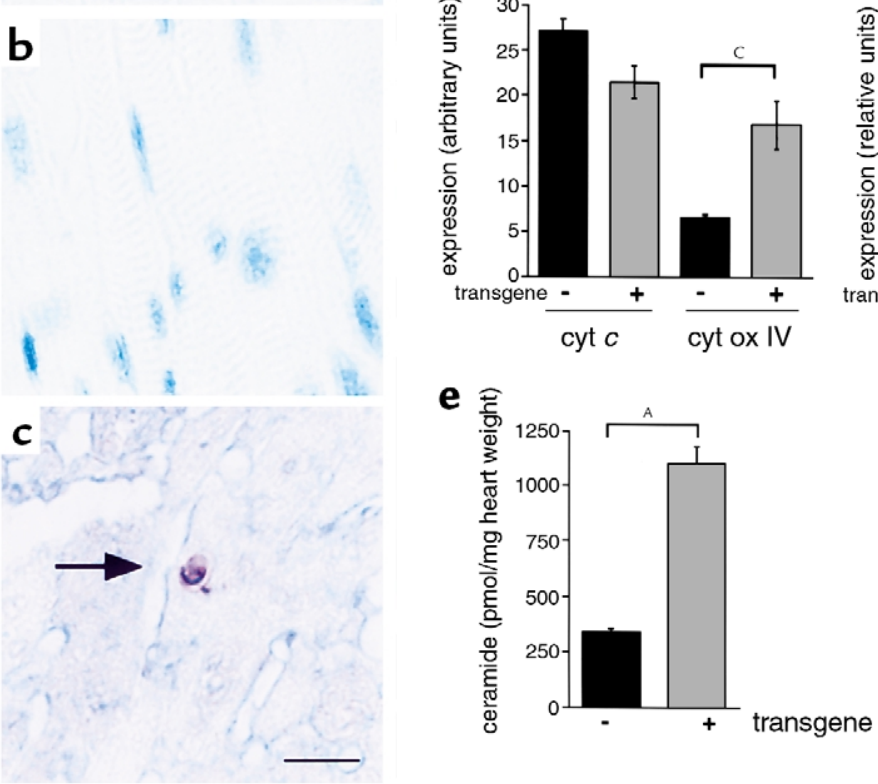

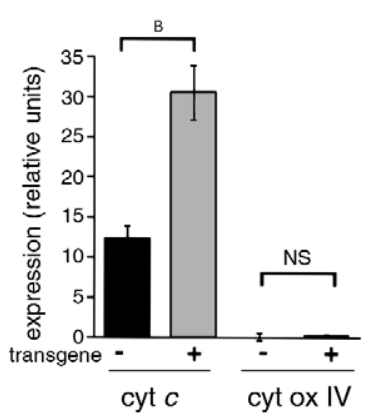

soluble fraction

- + transgene

cyt ox IV

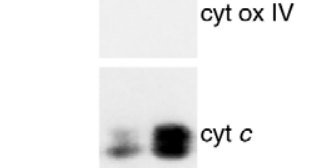

d

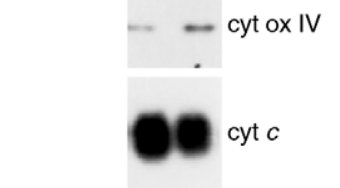




\section{Figure 8}

Light-microscopic examination of cardiac tissue from end-stage MHC-ACS O7 mice. Cardiac ventricular tissue was dissected from 28day-old MHC-ACS $\mathrm{O} 7$ animals $(\mathbf{a}, \mathbf{c}, \mathbf{e})$ and nontransgenic littermates $(\mathbf{b}, \mathbf{d}, \mathbf{f})$. Tissue was fixed in formalin, embedded in paraffin, and sectioned for H\&E staining $(\mathbf{a}, \mathbf{b})$ or Masson's trichrome $(\mathbf{e}, \mathbf{f})$. Tissue was flash-frozen and sectioned for oil red O staining (c, d). $\times 400$.

28-day-old mice were capable of metabolizing the excess triglyceride. By EM (Figure 9), surviving myocytes had marked loss of myofibrils, consistent with the vacuolated appearance by $\mathrm{H} \& \mathrm{E}$, and a paucity of lipid droplets, consistent with modest oil red $\mathrm{O}$ staining. In approximately $25 \%$ of cells, the cytoplasm also contained numerous, large, pleomorphic, abnormal organelles, consisting of complex multilamellar concentric membrane structures. These myelin body-like, membranous cytoplasmic bodies (MCBs) were not clearly derived from or contiguous with existing cellular organelles and were associated in some instances with the accumulation of excess membrane structures in interorganellar spaces. Biochemical analysis of lipid from hearts at this stage showed no significant differences between transgenic and wild-type mice in total triglyceride, cholesteryl ester, or glycerophospholipid content (data not shown). However, persistent evidence of acyl-chain remodeling was apparent with a decrease in the proportion of glycerophospholipids containing 22:6 at the $\mathrm{SN}-2$ position and increase in the proportion containing 18:1, 18:2, and 20:4.

\section{Discussion}

The MHC-ACS mice generated in the present study have markedly impaired cardiac lipid homeostasis with accumulation of triglyceride and phospholipids. These increases are observed in the setting of normal serum glucose and lipid levels and in the absence of underlying defects in myocardial $\beta$-oxidation. When followed longitudinally on a normal diet, MHC-ACS mice develop cardiac hypertrophy and heart failure, resulting in premature death. Thus, these mice serve as a model for metabolic cardiomyopathy in which the effects of lipid accumulation can be examined in isolation of primary defects in systemic glucose or fatty acid metabolism.

Lipid accumulation in MHC-ACS hearts is similar to that seen in human diseases and animal models of human disease. Perturbations of myocardial energy substrate utilization in both inherited and acquired cardiac diseases have been shown to result in lipid accumulation. Inborn errors in myocardial $\beta$-oxidation are associated with heart failure, arrhythmias, sudden death, and pathologic lipid accumulation with a threefold increase in phospholipids and 100-fold increase in triglycerides in the myocardium (32). Accumulation of long-chain fatty acyl intermediates and triglycerides have been reported in ischemia (17) and idiopathic dilated cardiomyopathy (33), respectively. Cardiac myocyte lipid accumulation may also contribute to the pathogenesis of diabetic cardiomyopathy. In animal models of diabetes, 2 - to 3.7-fold increases in myocyte triglyceride have

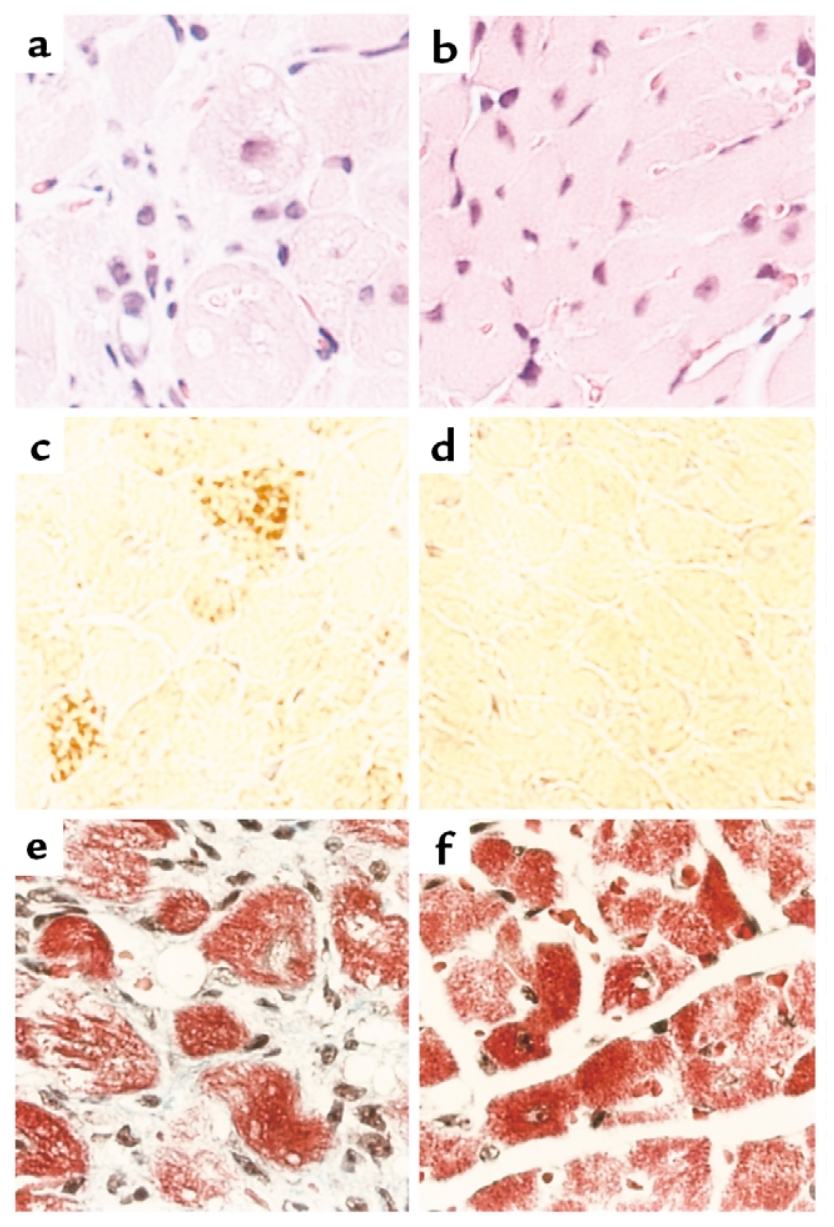

been reported $(20,34,35)$. Similarly, in obese Zucker diabetic fatty (ZDF) rats there is threefold increase in triglyceride accumulation, which is associated with cardiac dilatation and a small but significant reduction in leftventricular contractility (18). The MHC-ACS mice in this study have 12 -fold increase in cardiac triglyceride and $50 \%$ increase in cardiac choline glycerophospholipid content. The higher level of lipid accumulation in our model as compared with that observed in the ZDF rat likely contributes to more severe left-ventricular dysfunction in MHC-ACS mice. Nonetheless, the amount and biochemical composition of lipid accumulation in our model is similar to that seen in pathophysiologic conditions (32) and has relevance to human disease.

This study provides the first demonstration, in vivo, of ACS1 function in tissue lipid import. Cardiac myocyte lipid accumulation in MHC-ACS mice is consistent with a model in which ACS overexpression increases fatty acid import in excess of the capacity for $\beta$-oxidation and lipid degradation. These fatty acids are esterified to triglycerides and choline glycerophospholipids. ACS1 is one of several proteins whose expression facilitates uptake of fatty acids into mammalian cells in culture and into tissues in transgenic models. FATP $(9,36)$, CD36 $(37,38)$, and lipoprotein lipase (LPL) $(39,40)$ may function coordinately with ACS1 to regulate lipid homeostasis in mammalian tissues. These findings support the hypothesis that proteins per- 


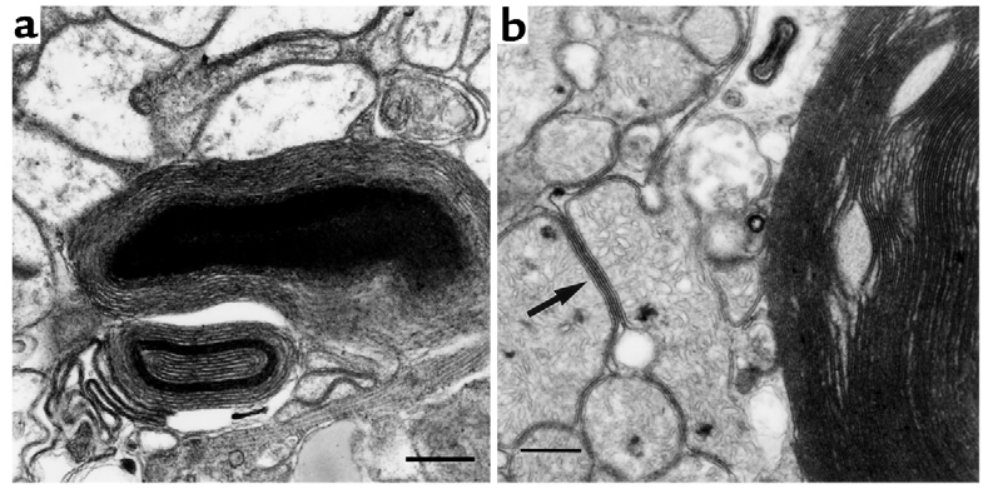

Figure 9

EM of cardiac tissue from end-stage MHC-ACS O7 mice. Ventricular tissue from a 28-day-old transgenic mouse was fixed in formalin and thin sectioned for EM. $\times 15,000$. Note complex structures composed of multiple layers of membrane in the cytoplasm of myocytes (a). In addition to these large multilamellar membrane aggregates, membranes often accumulated within the cytoplasm between organelles such as mitochondria (b) (arrow). Bar, $0.5 \mu \mathrm{m}$.

form an essential and/or regulatory role in fatty acid permeation into cells and are consistent with the general observations that molecules of physiological significance are transported across the plasma membrane by proteins rather than by passive diffusion (41).

Lipotoxicity has been observed in other transgenic and genetic models of altered lipid metabolism. Transgenic mice with muscle-specific overexpression of LPL have elevated muscle FFAs and develop a skeletal myopathy characterized by muscle fiber degeneration, fiber atrophy, glycogen storage, and extensive mitochondrial and peroxisomal proliferation (39). Neither mitochondrial nor peroxisomal proliferation was observed in MHCACS hearts. In the ZDF rat, triglyceride accumulation is associated with DNA laddering, suggesting that excess tissue fatty acids induce programmed cell death and lead to heart failure (18). Fatty acid-induced apoptosis has been observed previously in rat ventricular myocytes cultured in the presence of saturated but not monounsaturated fatty acids (42). Low millimolar concentrations of fatty acid initiate apoptosis in cultured pancreatic beta cells of ZDF rats and in hematopoietic (LyD9) cells by a mechanism involving de novo ceramide biosynthesis from palmitate (43-45) and subsequent activation of ceramide-signaling pathways (46). It is also possible that ceramide has direct pro-apoptotic effects on cardiac myocyte mitochondria (47).

The mechanism of cardiac myocyte loss and resultant heart failure in MHC-ACS mice likely involves both necrotic and apoptotic pathways. In nontransgenic hearts, there is no significant evidence for apoptosis as has been observed by others (48). In the MHC-ACS mice DNA fragmentation and cytochrome $c$ release are consistent with induction of apoptotic pathways. Although DNA fragmentation was observed in only a small percentage of myocytes, release of the proapoptotic cytochrome $c$ in this mitochondrial-rich tissue was readily detectable. In a previous study of apoptosis in heart failure, Narula and coworkers also noted a disparity between the easily detectable cytochrome $c$ release and rare evidence of in situ DNA fragmentation or apoptotic nuclear morphology (49). This paradox may be attributable to the cumulative significance of rare apoptotic events in a tissue composed of terminally differentiated cells. Moreover, relatively slow induction of apoptosis can lead to heart failure (50). In our model, lipid accumulation is associated with increased cardiac ceramide content. This signaling lipid may contribute to the induction or amplification of apoptotic pathways. On the other hand, findings of a mononuclear infiltrate and fibrosis at 28 days in end-stage MHC-ACS hearts suggests that in addition to apoptosis, some cell death occurs by necrosis in this model.

The histologic findings in MHC-ACS hearts suggest that cardiac myocytes are capable of channeling the excess lipid to additional distinct pathways. Initial observations show these cells are competent to form abundant lipid droplets, surrounded by discrete membrane(s). The spectrum of appearance of droplets with single to multiple surrounding membranes and the subsequent appearance of MCBs suggest that these initial amorphous droplets are metabolized to MCBs. This metabolism may be active or passive, since saturated and monounsaturated LCFA soaps in aqueous environments can spontaneously form bilayer structures (51). Alternatively, these structures may represent a membrane synthesis pathway that is activated to detoxify excess intracellular lipid. MCBs in MHC-ACS mice are similar to structures described in human diseases characterized by intracellular lipid accumulation, including Tay-Sachs and systemic late infantile lipidosis (52), Niemann-Pick type C (53), and adrenal gland tumors after treatment with spironolactone (spironolactone bodies) (54).

In summary, cardiac-restricted overexpression of ACS1 in our mouse model leads to marked cardiac myocyte lipid accumulation. This perturbation of lipid homeostasis results in the development of cardiomyopathy. MHC-ACS mice may serve as a model for the lipid-metabolic abnormalities in inherited and acquired human heart diseases, such as $\beta$-oxidation disorders and diabetic cardiomyopathy. Future studies of MHCACS mice will help elucidate cellular mechanisms of lipid toxicity and provide a model in which to test pharmacologic and genetic rescue strategies.

\section{Acknowledgments}

We are grateful to D. Kelly, K. Roth, T. Simons, D. Ory, and members of the Schaffer and Ory laboratories for helpful discussions. We acknowledge W. Kraft for assistance with electron microscopy, K. Green and K. Hutton for assistance with histology, C. Semenkovich and T. Coleman for serum analyses, and D. Thompson for lipid analysis. J. E. Schaffer is an Established Investigator of 
the American Heart Association. This work was supported by grants from the NIH (P50-HL-61006, P30-DK52574, P30-DK-56341, R01-HL-42665, RO1-DK-54268).

1. Neely, J.R., Rovetto, M.J., and Oram, J.F. 1972. Myocardial utilization of carbohydrate and lipids. Prog. Cardiovasc. Dis. 15:289-329.

2. van der Vusse, G.J., Glatz, J.F., Stam, H.C., and Reneman, R.S. 1992. Fatty acid homeostasis in the normoxic and ischemic heart. Physiol. Rev. 72:881-940.

3. Richieri, G.V., and Kleinfeld, A.M. 1995. Unbound free fatty acid levels in human serum. J. Lipid Res. 36:229-240.

4. Stremmel, W. 1988. Fatty acid uptake by isolated rat heart myocytes represents a carrier-mediated transport process. J. Clin. Invest. 81:844-852.

5. Sorrentino, D., Robinson, R.B., Kiang, C.-L., and Berk, P.D. 1989. At physiologic albumin/oleate concentrations oleate uptake by isolated hepatocytes, cardiac myocytes, and adipocytes is a saturable function of the unbound oleate concentration. J. Clin. Invest. 84:1325-1333.

6. Luiken, J.J.F.P., van Nieuwenhoven, F.A., America, G., van der Vusse, G.J., and Glatz, J.F.C. 1977. Uptake and metabolism of palmitate by isolated cardiac myocytes from adult rats: involvement of sarcolemmal proteins. J. Lipid Res. 38:745-758.

7. McArthur, M.J., et al. 1999. Cellular uptake and intracellular trafficking of long chain fatty acids. J. Lipid Res. 40:1371-1383.

8. Abumrad, N., Harmon, C., and Ibrahimi, A. 1998. Membrane transport of long-chain fatty acids: evidence for a facilitated process. J. Lipid Res. 39:2309-2318.

9. Schaffer, J.E., and Lodish, H.F. 1994. Expression cloning and characterization of a novel adipocyte long chain fatty acid transport protein. Cell. 79:427-436.

10. Gargiulo, C.E., Stuhlsatz-Krouper, S.M., and Schaffer, J.E. 1999. Localization of adipocyte long-chain fatty acyl-CoA synthetase at the plasma membrane. J. Lipid Res. 40:881-892.

11. Tanaka, T., Hosaka, K., Hoshimaru, M., and Numa, S. 1979. Purification and properties of long-chain acyl-CoA synthetase from rat liver. Eur. J. Biochem. 98:165-172.

12. Wittels, B., and Spann, J., Jr. 1968. Defective lipid metabolism in the failing heart. J. Clin. Invest. 47:1787-1794.

13. Bishop, S.P., and Altschuld, R.A. 1970. Increased glycolytic metabolism in cardiac hypertrophy and congestive failure. Am. J. Physiol. 218:153-159.

14. Christe, M.E., and Rodgers, R.L. 1994. Altered glucose and fatty acid oxidation in hearts of the spontaneously hypertensive rat. J. Mol. Cell. Cardiol. 26:1371-1375

15. Takeyama, D., et al. 1995. Effects of chronic right ventricular pressure overload on myocardial glucose and free fatty acid metabolism in the conscious rat. Cardiovasc. Res. 29:763-767.

16. Taegtmeyer, H., and Overturf, M.L. 1988. Effects of moderate hypertension on cardiac function and metabolism in the rabbit. Hypertension. 11:416-426.

17. Corr, P.B., Creer, M.H., Yamada, K.A., Saffitz, J.E., and Sobel, B.E. 1989. Prophylaxis of early ventricular fibrillation by inhibition of acylcarnitine accumulation. J. Clin. Invest. 83:927-936.

18. Zhou, Y.-T., et al. 2000. Lipotoxic heart disease in obese rats: implications for human obesity. Proc. Natl. Acad. Sci. USA. 97:1784-1789.

19. Avogaro, A., et al. 1990. Myocardial metabolism in insulin-deficient diabetic humans without coronary artery disease. Am. J. Physiol. 258:E606-E618

20. Kenno, D.A., and Severson, D.L. 1985. Lipolysis in isolated myocardial cells from diabetic rat hearts. Am. J. Physiol. 249:1024-1030.

21. Regan, T.J., and Weisse, A.B. 1992. Diabetic cardiomyopathy. J. Am. Coll. Cardiol. 19:1165-1166

22. Zoneraich, S., and Mollura, J.L. 1993. Diabetes and the heart: state of the art in the 1990s. Can. J. Cardiol. 9:293-299.

23. Fiordaliso, F., Latini, R., Li, B., Malhotra, A., and Kajstura, J. 1999. Myocyte loss in the diabetic heart is mediated by apoptosis. Circulation 100(Suppl. I):759.

24. Kelly, D.P., et al. 1992. Molecular basis of inherited medium-chain acylCoA dehydrogenase deficiency causing sudden child death. J. Inherit. Metab. Dis. 15:171-180.

25. Trippe, M.E., et al. 1981. Systemic carnitine deficiency presenting as familial endocardial fibroelastosis: a treatable cardiomyopathy. $N$. Engl. J. Med. 305:385-390.

26. Palermo, J., Gulick, J., Colbert, M., Fewell, J., and Robbins, J. 1996. Transgenic remodeling of the contractile apparatus in the mammalian heart. Circ. Res. 78:504-509.

27. Rogers, J.H., et al. 1999. RGS4 causes increased mortality and reduced cardiac hypertrophy in response to pressure overload. J. Clin. Invest. 104:567-576.

28. Bligh, E.G., and Dyer, W.J. 1959. A rapid method of total lipid extraction and purification. Canadian Journal of Biochemistry and Physiology. 37:911-917

29. Han, X., Gubitosi-Klug, R.A., Collins, B.J., and Gross, R.W. 1996. Alterations in individual molecular species of human platelet phospholipids during thrombin stimulation: electrospray ionization mass spectrometry-facilitated identification of the boundary conditions for the magnitude and selectivity of thrombin-induced platelet phospholipid hydrolysis. Biochemistry. 35:5822-5832.

30. Van Veldhoven, P.P., Bishop, W.R., Yurivich, D.A., and Bell, R.M. 1995. Ceramide quantitation: evaluation of a mixed micellar assay using E. col diacylglycerol kinase. Biochem. Mol. Biol. Int. 36:21-30.

31. Desagher, S., et al. 1999. Bid-induced conformational change of bax is responsible for mitochondrial cytochrome c release during apoptosis. $J$. Cell Biol. 144:891-901.

32. Galloway, J.H., Cartwright, I.J., and Bennett, M.J. 1987. Abnormal myocardial lipid composition in an infant with type II glutaric aciduria. J. Lipid Res. 28:279-284.

33. Tomita, T., Wilson, L., and Chiga, M. 1990. Idiopathic dilated cardiomyopathy: an evidence of abnormal lipid accumulation in myocardium. Am. J. Cardiovasc. Pathol. 3:81-85.

34. Denton, R., and Randle, P. 1967. Concentrations of glycerides and phospholipids in rat heart and gastrocnemius muscles: effects of alloxan-diabetes and perfusion. Biochem. J. 104:416-422.

35. Paulson, D.J., and Crass, M.F.I. 1982. Endogenous triacylglycerol metabolism in diabetic heart. Am. J. Physiol. 242:H1084-H1094.

36. Stahl, A., et al. 1999. Identification of the major intestinal fatty acid transport protein. Mol. Cell. 4:299-308.

37. Febbraio, M., et al. 1999. A null mutation in murine CD36 reveals an important role in fatty acid and lipoprotein metabolism. J. Biol. Chem. 274:19055-19062.

38. Ibrahimi, A., et al. 1999. Muscle-specific overexpression of FAT/CD36 enhances fatty acid oxidation by contracting muscle, reduces plasma triglycerides and fatty acids, and increases plasma glucose and insulin. J. Biol. Chem. 274:26761-26766.

39. Levak-frank, S., et al. 1995. Muscle-specific overexpression of lipoprotein lipase causes a severe myopathy characterized by proliferation of mitochondria and peroxisomes in transgenic mice. J. Clin. Invest. 96:976-986.

40. Weinstock, P., et al. 1995. Severe hypertriglyceridemia, reduced high density lipoprotein, and neonatal death in lipoprotein lipase knockout mice. Mild hypertriglyceridemia with impaired very low density lipoprotein clearance in heterozygotes. J. Clin. Invest. 96:2555-2568.

41. Al-Awqati, Q. 1999. One hundred years of membrane permeability: does Overton still rule? Nat. Cell Biol. 1:E201-E202.

42. deVries, J.E., et al. 1997. Saturated but not mono-unsaturated fatty acids induce apoptotic cell death in neonatal rat ventricular myocytes. J. Lipid Res. 38:1384-1394.

43. Paumen, M.B., Ishida, Y., Muramatsu, M., Yamamoto, M., and Honjo, T. 1997. Inhibition of carnitine palmitoyltransferase I augments sphingolipid synthesis and palmitate-induced apoptosis. J. Biol. Chem. 272:3324-3329.

44. Shimabukuro, M., et al. 1998. Lipoapoptosis in beta-cells of obese prediabetic fa/fa rats. J. Biol. Chem. 273:32487-32490.

45. Shimabukuro, M., Zhou, Y.T., Levi, M., and Unger, R.H. 1998. Fatty acidinduced B cell apoptosis: a link between obesity and diabetes. Proc. Natl. Acad. Sci. USA. 95:2498-2502.

46. Mathias, S., Pena, L.A., and Kolesnick, R.N. 1998. Signal transduction of stress via ceramide. Biochem. J. 335:465-480.

47. Paola, M.D., Cocco, T., and Lorusso, M. 2000. Ceramide interaction with the respiratory chain of heart mitochondria. Biochemistry. 39:6660-6668.

48. Kang, P.M., and Izumo, S. 2000. Apoptosis and heart failure. Circ. Res. 86:1107-1113.

49. Narula, J., et al. 1999. Apoptosis in heart failure: release of cytochrome c from mitochondria and activation of caspase- 3 in human cardiomyopathy. Proc. Natl. Acad. Sci. USA. 96:8144-8149.

50. Wencker, D., et al. 1999. Myocyte apoptosis is sufficient to cause dilated cardiomyopathy. Circulation. 100(Suppl. I):17

51. Cistola, D.P., Atkinson, D., Hamilton, J.A., and Small, D.M. 1986. Phase behavior and bilayer properties of fatty acids: hydrated 1:1 acid-soaps. Biochemistry. 25:2804-2812.

52. Suzuki, K., Suzuki, K., and Chen, G.C. 1968. Morphological, histochemical and biochemical studies on a case of systemic late infantile lipidosis (generalized gangliosidosis). J. Neuropathol. Exp. Neurol. 1:15-38.

53. Blanchette-Mackie, E.J., et al. 1988. Type-C Niemann-Pick disease: low density lipoprotein uptake is associated with premature cholesterol accumulation in the Golgi complex and excessive cholesterol storage in lysosomes. Proc. Natl. Acad. Sci. USA. 85:8022-8026.

54. Janigan, D.T. 1963. Cytoplasmic bodies in the adrenal cortex of patients treated with spironolactone. Lancet. 1:850-852. 